\title{
The pathogenic role of the adaptive immune response to modified LDL in diabetes
}

\section{Gabriel Virella ${ }^{1}$ and Maria F. Lopes-Virella $a^{1,2}$}

${ }^{1}$ Department of Microbiology and Immunology, Medical University of South Carolina, Charleston, SC, USA

${ }^{2}$ Ralph E. Johnson VA Medical Center, Charleston, SC, USA

\section{Edited by:}

Charles M. Alexander, Merck, USA

Reviewed by:

Shinichi Oikawa, Nippon Medical

School, Japan

James Whitfield Reed, Morehouse

School of Medicine, USA

\section{*Correspondence:}

Gabriel Virella, Department of Microbiology and Immunology,

Medical University of South Carolina,

173 Ashley Avenue, MSC 504,

Charleston, SC 29425-5040, USA.

e-mail: virellag@musc.edu
The main causes of morbidity and mortality in diabetes are macro and microvascular complications, including atherosclerosis, nephropathy, and retinopathy. As the definition of atherosclerosis as a chronic inflammatory disease became widely accepted, it became important to define the triggers of vascular inflammation. Oxidative and other modifications of lipids and lipoproteins emerged as major pathogenic factors in atherosclerosis. Modified forms of $L D L(m L D L)$ are pro-inflammatory by themselves, but, in addition, mLDLs including oxidized, malondialdehyde (MDA)-modified, and advanced glycation end (AGE)-product-modified LDL induce autoimmune responses in humans. The autoimmune response involves $T$ cells in the arterial wall and synthesis of $\lg G$ antibodies. The $\lg G$ auto-antibodies that react with $\mathrm{mLDLs}$ generate immune complexes (IC) both intra and extravascularly, and those IC activate the complement system as well as phagocytic cells via the ligation of Fc $\gamma$ receptors. In vitro studies proved that the pro-inflammatory activity of IC containing $\mathrm{mLDL}(\mathrm{mLDL}-\mathrm{IC})$ is several-fold higher than that of the modified LDL molecules. Clinical studies support the pathogenic role of $\mathrm{mLDLIC}$ in the development of macrovascular disease patients with diabetes. In type 1 diabetes, high levels of oxidized and AGE-LDL in IC were associated with internal carotid intima-media thickening and coronary calcification. In type 2 diabetes, high levels of MDA-LDL in IC predicted the occurrence of myocardial infarction. There is also evidence that $\mathrm{mLDL-IC}$ are involved in the pathogenesis of diabetic nephropathy and retinopathy. The pathogenic role of $\mathrm{mLDLIC}$ is not unique to diabetic patients, because those IC are also detected in non-diabetic individuals. But $\mathrm{mLDLIC}$ are likely to reach higher concentrations and have a more prominent pathogenic role in diabetes due to increased antigenic load secondary to high oxidative stress and to enhanced autoimmune responses in type 1 diabetes.

Keywords: LDL modification in diabetes, immune complexes, autoimmune response to modified LDL, diabetic complications, oxidized LDL, oxidized LDL antibodies, atherosclerosis, nephropathy

\section{INTRODUCTION: LDL MODIFICATION}

Oxidative stress is believed to be a critical factor in the initiation of pathogenic pathways that lead to the development of complications in diabetes (Giacco and Brownlee, 2010). Hyperglycemia plays a key role by inducing mitochondrial overproduction of reactive oxygen species (ROS), which, in turn, will cause oxidative modification of proteins, enzymes, and other substrates, including the formation of advanced glycation end (AGE) products (Giacco and Brownlee, 2010; Miller et al., 2010).

Lipoproteins are among the proteins that are modified as a consequence of oxidation and glycation. Endothelial cells (EC), monocytes/macrophages, lymphocytes, and smooth muscle cells (SMC) are all able to enhance the rate of oxidation of LDL. ROS and sulfur-centered radicals initiate metal ion-dependent lipid peroxidation leading to the generation of aldehydes that interact with lysine residues in ApoB-100, resulting in oxidation of LDL. Alternatively, endothelial injury secondary to oxidative stress results in increased prostaglandin synthesis and platelet activation. These processes also cause the formation of aldehydes such as malondialdehyde (MDA) that interact with the lysine residues of ApoB-100 (Holvoet, 1999). In vitro, MDA-lysine (as well as carboxymethyl lysine, carboxyethyl lysine, and other unidentified modifications) are generated by copper oxidation of LDL. Direct treatment of LDL with MDA, on the other hand, results in the formation of highly modified LDL with a 10-fold excess of MDAlysine over copper-oxidized LDL (oxLDL) and no other detectable modifications (Virella et al., 2004).

\section{PATHOGENIC ROLE OF MODIFIED LDL}

The pathogenic role of modified lipoproteins in the progression of atherosclerosis is well established. It has been investigated from two different angles: the direct pro-atherogenic effect of modified forms of LDL (mLDL; Lopes-Virella and Virella, 2003; Miller et al., 2010) and the consequences of the immune response directed against neoepitopes resulting from lipoprotein modification (Lopes-Virella and Virella, 2010). Both types of effects have been extensively characterized in the case of oxLDL. OxLDL is taken up by macrophages via receptor-mediated pathways other than the classic LDL receptor (Henriksen et al., 1983; Arai et al., 1989; Sparrow et al., 1989; Endemann et al., 1993; Penn and Chisolm, 
1994) and it induces accumulation of cholesteryl esters and the transformation of macrophages into foam cells (Fogelman et al., 1980; Hoff et al., 1989). It has also been reported that high concentrations of oxLDL are cytotoxic and experimental data suggests that oxLDL can injure vascular cells, both endothelial and SMC (Henriksen et al., 1979; Hessler et al., 1983). Furthermore, oxLDL induces enhanced synthesis of growth factors, including PDGF-AA, and PDGF receptor in SMC, as well as of granulocytemonocyte colony stimulating factor, macrophage colony stimulating factor, and granulocyte-colony stimulating factor in aortic EC from humans and rabbits (Rajavashisth et al., 1990). In addition, oxLDL may affect fibrinolysis, by inhibiting the secretion of tissue plasminogen activator (tPA) by human EC (Kugiyama et al., 1993) and stimulating the secretion of plasminogen activator inhibitor (PAI)-1 (Kugiyama et al., 1993). Thus, oxLDL inhibits the endothelium-dependent activation of fibrinolysis, possibly promoting a chronic prothrombotic state.

Oxidized LDL has also been found to have pro-inflammatory effects relevant to the atherosclerotic process. It has chemotactic effects on monocytes (Quinn et al., 1987), enhances monocyte adhesion to EC in culture (Berliner et al., 1990; Kume et al., 1992), as well as the expression of VCAM-1 and ICAM-1 by human aortic EC induced by TNF $\alpha$ (Kahn et al., 1995) and of ICAM-1 in resting human endothelial vein cells (Takei et al., 2001). These pro-inflammatory effects are the result of the activation of a variety of functional pathways intimately related to innate immunity processes (Shalhoub et al., 2011). Finally, oxLDL has been shown to activate a variety of cell types expressing CD36 and other scavenger receptors and contribute to the generation of ROS (Li et al., 2010).

Advanced glycation end-product-modified LDL, as well as other AGE-modified proteins, are also pro-inflammatory (Vlassara et al., 2002; Wendt et al., 2002). AGE-modified proteins will impact EC eliciting increased permeability and pro-coagulant activity (Vlassara et al., 1994) and inducing the overexpression of VCAM-1 (Schmidt et al., 1995). AGE also contributes to fibroblast proliferation and T cell activation (Vlassara et al., 1994), and activated $\mathrm{T}$ cells in the atheromatous lesions release interferon- $\gamma$ (De Boer et al., 1999), which in turn will prime macrophages in the lesion enhancing the release of pro-inflammatory cytokines and chemotactic factors in response to the recognition of AGE-LDL and other mLDL by the corresponding receptors. The impact of AGE in the atherosclerotic process associated with diabetes was confirmed in streptomycin-induced diabetic ApoE ${ }^{-1-}$ mice. Administration of soluble forms of AGE receptors (RAGE) resulted in reduction of vascular permeability and slowed down the progression of atheromatous lesions (Bucciarelli et al., 2002).

\section{THE IMMUNOGENICITY OF MODIFIED LDL}

The pro-inflammatory properties of modified LDL appear to be considerably enhanced as a consequence of their immunogenicity. The immunogenicity of modified LDL was first reported by Steinbrecher et al. (1984) based on the immunization of laboratory animals with several types of modified LDL. Of all the mLDL, oxLDL has been studied in greatest detail from the immunological point of view. Steinbrecher (1987) as well as Palinski et al. (1990) characterized its immunogenic epitopes. Furthermore, human auto-antibodies to oxLDL were the first to be purified and characterized (Yla-Herttuala et al., 1994; Mironova et al., 1996; Virella et al., 2000). The cell-mediated immune system is also activated by antigen-presenting cells presenting modified LDL oligopeptides together with co-stimulatory signals to Th-1 cells, resulting in a chronic inflammatory reaction in which interferon$\gamma$ released by Th- 1 cells enhances the pro-inflammatory response of macrophages, including the release of chemokines that attract more $\mathrm{T}$ cells to the area and the process becomes self-perpetuating (De Boer et al., 1999; Andersson et al., 2010).

\section{CELLULAR RESPONSE TO ANTIGEN-ANTIBODY COMPLEXES (IMMUNE COMPLEXES) CONTAINING DIFFERENT MODIFIED FORIMS OF LDL}

It has been established that atherosclerotic plaque rupture is a critical event triggering thrombus formation and subsequent acute coronary events (Libby and Theroux, 2005). Plaques that are prone to rupture consist of a larger intimal lesion with abundant macrophages and foam cells and a thinned fibrous cap (Shah, 2002). Necropsy studies have demonstrated that atherosclerosis in diabetic patients is more diffuse and accelerated than in nondiabetic patients (Jarrett, 1981). Furthermore, studies have also shown that atherosclerotic lesions in diabetic patients were more vulnerable as they had larger intimal lesions and more macrophage infiltration as compared to those in non-diabetic patients (Moreno et al., 2000). Analysis of gene expression in atherosclerotic plaques showed that when compared to stable plaques, vulnerable plaques have higher expression of matrix metalloproteinases (MMPs) with collagenase activity, which contribute to the thinning of the fibrous cap, causing plaque instability and rupture (Galis et al., 1994). Among the MMPs, MMP-9 has been the object of considerable interest in recent years, and according to some studies is an independent risk factor for atherothrombotic events (Loftus et al., 2001; Blankenberg et al., 2003). MMP-9 synthesis and release can be induced through TLR-4 stimulation, usually involving bacterial endotoxins (Lundberg and Hansson, 2010), but also by minimally modified LDL (Choi et al., 2009) and likely by other types of modified LDL.

Besides overexpression of MMPs, vulnerable plaques are characterized by the accumulation of apoptotic macrophages around the necrotic core (Seimon and Tabas, 2009). A variety of proapoptotic insults has been proposed to play a significant role in the evolution of atheromas, including oxidative stress, endoplasmic reticulum (ER) stress, accumulation of non-esterified (free) cholesterol, and effects of pro-inflammatory cytokines released by activated macrophages (Seimon and Tabas, 2009). Most likely these factors play additive or synergistic effects in the induction of apoptosis. For example, intracellular accumulation of free cholesterol is a known inducer of ER stress, but low levels of ER stress usually protect against apoptosis (Seimon and Tabas, 2009). On the other hand, accumulation of free cholesterol in macrophages in combination with signals delivered through scavenger receptors or with interferon- $\gamma$, known to be released by activated $\mathrm{T}$ cells in atheromas (De Boer et al., 1999; de Boer et al., 2000), leads to serine phosphorylation of STAT-1 which is a critical element in the induction of apoptosis secondary to ER stress (Lim et al., 2008). The apoptotic macrophages in atheromas are ingested 
by functional macrophages (efferocytosis). Efferocytosis in early lesions seems to result in suppression of inflammation, while in advanced lesions is associated with enhanced inflammation (Seimon and Tabas, 2009). This evolution appears to be the result of defective efferocytosis in advanced lesions, allowing the apoptotic cells to undergo necrosis, resulting in the accumulation of cell fragments that promote inflammation and plaque instability (Seimon and Tabas, 2009).

The activation of functional pathways by oxLDL and immune complex (IC) containing oxLDL has been studied in detail. oxLDL has been shown to activate a variety of cell types expressing CD36 and other scavenger receptors and contribute to the generation of ROS (Li et al., 2010). On macrophages, the interaction of oxLDL with CD36 (mediated by oxidized phospholipids) results in activation of the src family members Fyn/Lyn, and of several components of the MAP kinase pathway, including MKKK, MKK, FAK, and mitogen-activated protein kinase (MAPK; c-Jun N-terminal kinase, c-JNK; Silverstein et al., 2010). The activation of these kinases and associated proteins such as Vav is associated with foam cell formation as well as with unregulated actin polymerization and loss of cell polarity causing a migration defect and the trapping of activated cells in the atheromatous lesions (Silverstein et al., 2010). In platelets the same signaling events lead to enhanced platelet reactivity and enhanced formation of thrombi (Silverstein, 2009). Recently it has been reported that ligation of CD36 by oxLDL leads to the formation of a TLR-4-TLR- 6 heterodimer that, in turn, will activate MyD88 and NFkB, a critical step in the induction of the synthesis and release of pro-inflammatory cytokines (Stewart et al., 2010).

OxLDL-IC have been demonstrated to be more potent activators of human macrophages than oxLDL (Saad et al., 2006). The uptake of IC prepared with native or oxLDL by human macrophages is primarily mediated by Fc $\gamma$ receptors, primarily Fc $\gamma$ RI (Lopes-Virella et al., 1991, 1997; Oksjoki et al., 2006). It has been shown that binding of IgG antibody to oxLDL blocks the interaction of oxLDL with CD36 (Nagarajan, 2007), so CD36 is not involved in the process. For MDA-LDL-IC and AGE-LDL-IC $\mathrm{Fc} \gamma \mathrm{RI}$ is also involved, but the possible interaction of these $\mathrm{mLDL}$ with scavenger receptors or receptors for AGE-modified proteins has not been proven or excluded.

One fundamental property of modified LDL-IC is their ability to deliver large concentrations of free and esterified cholesterol to macrophages (Virella et al., 2002). The intracellular accumulation of cholesterol by itself may not induce apoptosis (Seimon and Tabas, 2009). In fact, both oxLDL-IC and oxLDL (at concentrations not exceeding $75 \mu \mathrm{g} / \mathrm{mL}$ ) have the opposite effect and prevent macrophage apoptosis (Hundal et al., 2003; Oksjoki et al., 2006). In vitro data suggests that oxLDL-IC have a predominantly anti-apoptotic effect, more pronounced than that of oxLDL (Hammad et al., 2006; Oksjoki et al., 2006) but not unique to oxLDL-IC, because it has also been reproduced with keyhole limpet hemocyanin (KLH)-anti-KLH-IC (Oksjoki et al., 2006). However, there are significant differences between oxLDL-IC and other IgG-containing IC. Only oxLDL-IC can both engage Fc $\gamma$ RI and deliver cholesterol to the cells and the magnitude of the proinflammatory response induced in human macrophages is greater with oxLDL-IC than with KLH-IC, for example Saad et al. (2006).
While oxLDL cell signaling is mediated by scavenger receptors, oxLDL-IC deliver activating signals via Fc $\gamma$ receptors. The crosslinking of Fc $\gamma$ receptors by IC induces phosphorylation of ITAMs by kinases of the Src family, and consequent activation of Syk (Crowley et al., 1997; Tohyama and Yamamura, 2009). Activation of Syk triggers a variety of pathways, including the MAPK signaling cascade, which includes ERK1/2, p38 MAPK, and c-JNK (Luo et al., 2010), responsible for NFkB activation and the expression of pro-inflammatory gene products, and the PI3K and AKT pathway secondary to phospholipase C activation (Oksjoki et al., 2006), which promotes cell survival by at least four different mechanisms: (1) phosphorylating the Bad component of the $\mathrm{Bad} / \mathrm{Bcl}-\mathrm{X}_{\mathrm{L}}$ complex which results in its dissociation and cell survival, (2) caspase 9 inactivation, (3) regulation of the expression of transcription factors, and (4) activation of IKK kinases which phosphorylate $\mathrm{I} \kappa \mathrm{B}$ and, as a consequence, release the active form of NFkB which upregulates the expression of genes favoring cell survival (Datta et al., 1999).

Furthermore, the anti-apoptotic effect of oxLDL-IC seems to involve additional pathways, including activation of sphingosine kinase 1, which causes the levels of anti-apoptotic sphingosine1-phosphate (S1P) to increase. S1P activates phospholipase C (PLC) and, through the generation of diacylglycerol, the Ras/ERK, and phosphokinase $\mathrm{C}$ are activated. PLC also activates the P13Kdependent pathway, which results in Akt activation (Hundal et al., 2003; Hammad et al., 2006; Chen et al., 2010; Figure 1).

Not surprisingly, the repertoire of oxLDL-IC-induced prosurvival genes is much wider than that induced by oxLDL alone (Hammad et al., 2009). Also, oxLDL-IC induce HSP70B expression in macrophages. This protein binds to the internalized lipid moiety of oxLDL-IC and prevents its degradation, while at the same time inducing sphingokinase-1 (Al Gadban et al., 2010; Smith et al., 2010).

In contrast to oxLDL, there is no published information concerning pathways of cell activation triggered by MDA-LDL or MDA-LDL-IC. The association of MDA-LDL with acute coronary syndromes (Holvoet et al., 1998; Holvoet, 1999) and the association of high levels of MDA-LDL in the circulating IC isolated from patients with type 2 diabetes who had acute CVD events, mainly MI (discussed later in this review), strongly suggest that MDALDL and MDA-LDL-IC have pro-apoptotic activity, although the precise pathways involved can only be suggested (Figure 1). Preliminary results obtained in our laboratory in experiments exposing human monocyte-derived macrophages to MDA-LDL-IC have shown increased expression of caspase 3, implying that, in contrast to oxLDL-IC, MDA-LDL-IC do not activate survival pathways. This difference between oxLDL and MDA-LDL could be a result of the large excess of MDA-modified lysine molecules in MDA-LDL relative to oxLDL (Virella et al., 2005). Also, while copper oxidation predominantly results in ApoB fragmentation, MDA modification is associated with ApoB aggregation (Viita et al., 1999). Obviously, physico-chemical differences in ApoB could determine different biological properties of the two forms of modified LDL.

Modified LDL isolated from circulating IC reacts with antibodies to oxidized, MDA, and AGE-modified LDL. The content of these modifications in IC-associated LDL is variable from patient to patient, but overall it reflects the predominance of a given type of 


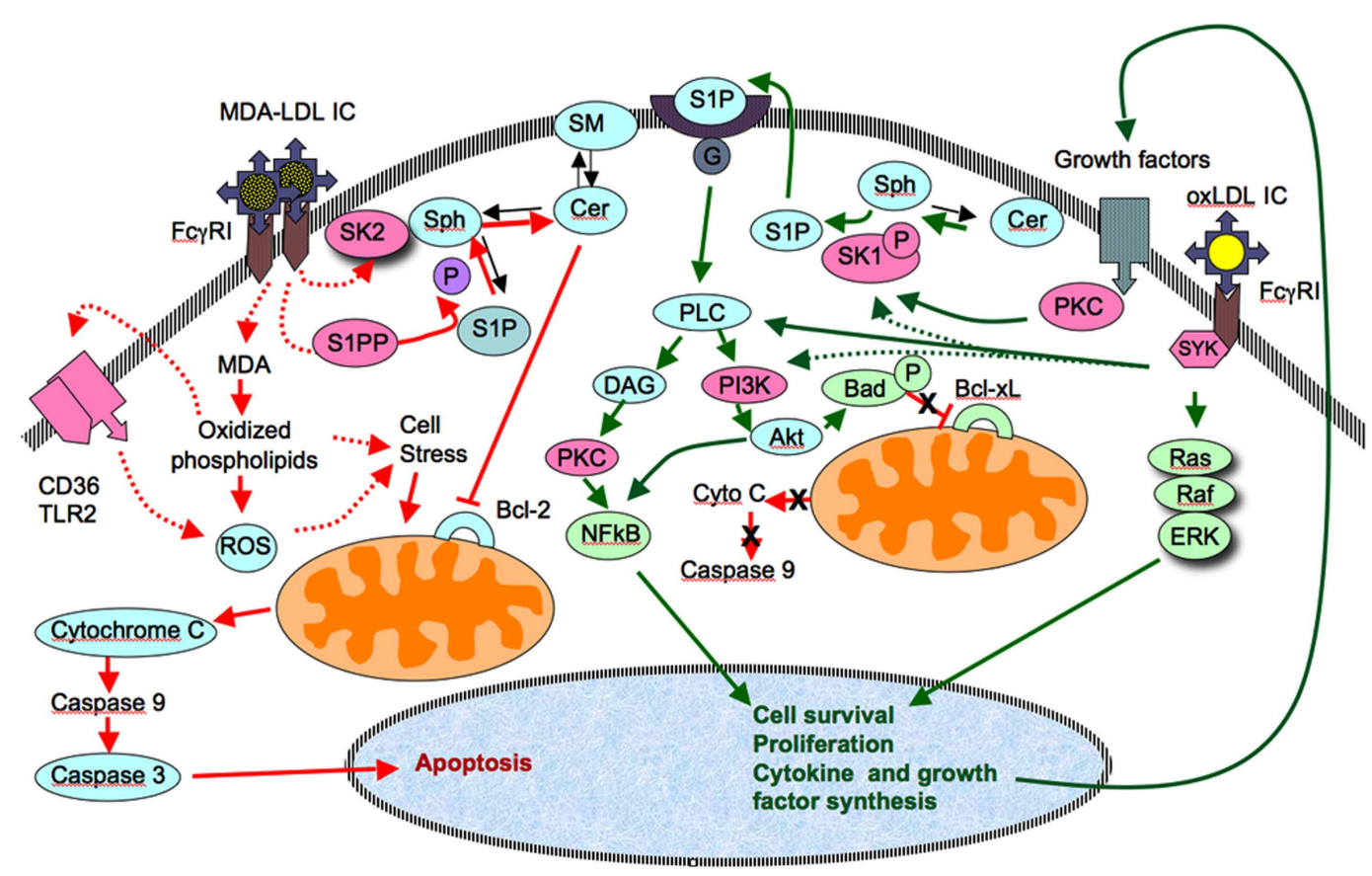

FIGURE 1 | Comparison of the pathways responsible for the anti-apoptotic and pro-apoptotic effects of immune complexes containing oxidized LDL (oxLDL-IC) and malondialdehyde-modified LDL (MDA-LDL-IC). OxLDL-IC activate cell proliferation pathways through Syk, a pathway that leads to activation of Akt and NFkB. The activation of Akt leads prevents the inactivation of anti-apototic gene products (Bcl-xL in the diagram). S1P-mediated activation of Akt and proliferation genes has been suggested by previously published data from our group (Hammad et al., 2006). This could result from the direct activation of SK1 by Syk, or as a consequence of the release of growth factors, upon ligation of the corresponding receptor, which activate S1k via PKC. As for the pro-apoptotic properties of MDA-LDLIC, two possible pathways could be involved. One would result from the simultaneous activation of SK2 (whose phosphorylation is less stable than that of SK1) and S1PP. This would result in a reduced generation of S1P, and accumulation of ceramides, which in turn would inhibit anti-apoptotic genes (Bcl-2 in the diagram) and allow the activation of the pro-apoptotic intrinsic pathway. An alternative (and not exclusive) pathway to reach the same effect would involve the degradation of internalized MDA and release of highly charged phospholipids whose interaction with a CD36-TLR2 complex would activate the generation of ROS and increased cellular stress. epitope. The enrichment in MDA-modified lysine could explain the differences in clinical associations that emerged in the data obtained in the EDIC/DCCT cohort and the VADT patient cohort. In the EDIC/DCCT cohort, high levels of oxLDL (which in vitro experiments show that is associated with macrophage survival) (Oksjoki et al., 2006; Hammad et al., 2009) in isolated IC are strong predictors of progression of CAD, as assessed by longitudinal measurements of carotid intima-media thickness (IMT; Lopes-Virella et al., 2011a). In contrast, the levels of MDA-LDL (which in vitro data show that is associated with macrophage apoptosis) in isolated IC, although associated with CAD progression, are a weaker predictor, comparable to high levels of LDL-cholesterol (LopesVirella et al., 2011a). This could be a reflection of the fact that at the time of admission into the DCCT/EDIC cohort the patients were young and basically CAD-free. Therefore, at that stage, the pro-apoptotic effect of MDA-LDL-IC would be associated with effective efferocytosis and the inhibition of the expansion of atherosclerotic lesions (Seimon and Tabas, 2009) However, in older patients with more advanced lesions, like those included in the VADT cohort, chronic and extensive ER stress is present leading to impaired processing of heavily oxidized and aggregated LDL by macrophages as well as to defective efferocytosis (Hoff et al., 1993). Defective efferocytosis favors macrophage necrosis and the release of pro-inflammatory mediators and MMPs leading to plaque destabilization. Release of cholesterol and oxidized phospholipids also takes place when macrophages undergo necrosis and the oxidized phospholipids can be transported to the extracellular compartment and then react with scavenger receptors and/or TLRs, delivering signals that favor the activation of pro-apoptotic pathways, increase cellular stress, and block the anti-apoptotic pathways. Recent data from our laboratory, still unpublished, supports the pro-apoptotic effect of MDA-LDL-IC in human macrophages and data obtained in the VADT cohort supports the role of MDA-LDL-IC in inducing plaque destabilization and acute CVD events (Lopes-Virella et al., 2012b).

\section{MODIFIED LDL CONCENTRATIONS AS RISK FACTORS FOR DIABETIC COMPLICATIONS}

Our group has studied extensively the pathogenic role of modified LDL antibodies (Virella et al., 2002, 2008; Saad et al., 2006; LopesVirella et al., 2007, 2011a; Lopes-Virella and Virella, 2010), and has developed methodology for the measurement of circulating antibodies to mLDL (Virella et al., 1993) and for the measurement of modified form of LDL and the corresponding antibodies involved in IC formation through the isolation and fractionation of circulating IC (Atchley et al., 2002; Virella et al., 2004, 2005, 
2008). Several groups reported studies concerning the possible association between modified LDL (particularly oxLDL) or the corresponding antibodies with cardiovascular disease with conflicting results (Virella and Lopes-Virella, 2003; Lopes-Virella and Virella, 2010). The proposed assays for modified LDL are mostly enzymoimmunoassays and are affected by fact that $95 \%$ of circulating modified LDL exists as part of IC (Virella et al., 2004), a well-known cause of error in both antigen and antibody assays. The interference of IC was never clearly addressed in the assays used by different groups, and there has been no effort to develop a standard assay that could accurately measure circulating forms of modified LDL. Orekhov et al. (1991, 1995) first called attention to the use of circulating IC containing LDL as markers indicative of the severity of the atherosclerotic process. Their methodology consisted of precipitating IC from serum samples and measuring the cholesterol content in the precipitates as a surrogate marker for LDL. We used a similar approach in our initial attempts to measure LDL-IC levels (Lopes-Virella et al., 2007), but we decided that a direct measurement of modified LDL in precipitated IC would be more specific and informative. Our current approach, as previously noted, involves isolation and fractionation of circulating IC and allows to measure the levels of different forms of modified LDL involved in IC formation without interference of the high affinity IgG auto-antibodies (Virella et al., 2005). The composition of those IC should reflect that of the complexes deposited in the arterial wall, given that both LDL and IgG antibodies can diffuse across the endothelial barrier (Langer et al., 1972; Virella, 2007).

Data generated in clinical studies carried out on a type 1 diabetes cohort (the DCCT/EDIC cohort) have shown that high levels of oxLDL and AGE-LDL in circulating IC are associated with increased odds to develop diabetic nephropathy (Lopes-Virella et al., 2012a) and progression of retinopathy (Lopes-Virella et al., 2012c). Also in nephropathy, predominance of IgG antibodies (particularly those with higher avidity) over IgM antibodies in oxLDL-IC was associated with parameters indicative of deteriorating renal function in same cohort (Atchley et al., 2002; Virella et al., 2008). Using coronary artery calcification (CAC) indices and carotid IMT as end-points indicative of cardiovascular disease progression we also found that increased levels of oxLDL and of AGE-LDL in circulating IC are associated in the DCCT/EDIC cohort with the development of coronary calcification and with increased levels and progression of carotid IMT. The levels of MDA-LDL in isolated IC show a significant but weaker correlation with increased carotid IMT (Lopes-Virella et al., 2011a,b). In contrast, in patients with type 2 diabetes (VADT cohort), the levels of oxLDL and AGE-LDL in circulating IC are not significantly associated with the occurrence of acute events but high concentrations of MDA-LDL in IC are strong predictors of acute events, especially myocardial infarction (Lopes-Virella et al., 2012b). It must be noted that Holvoet et al. $(1995,1998)$ reported in two separate studies a link between high levels of oxLDL and established CAD and between elevated plasma MDA-LDL levels and plaque instability. As previously discussed, the association of circulating MDA-LDL and IC-associated MDA-LDL specifically with plaque instability/acute $\mathrm{CV}$ events raises interesting questions such as whether differences in the predominant species of modified
LDL involved in IC formation may induce distinct cell signaling patterns and, in the case of IC carrying predominantly MDALDL, lead to plaque instability by inducing macrophage apoptosis and/or increased synthesis of MMPs, such as MMP-9 (Koenig and Khuseyinova, 2007), known to break down collagen and thus contribute to plaque thinning and rupture. This is very novel concept, proposing that the effects of the interaction of IC with phagocytic and antigen-presenting cells depend not only of the engagement of $\mathrm{Fc} \gamma$ receptors but also of the physico-chemical characteristics of the antigen.

One significant question that has not yet been directly answered is whether the formation and pathogenic role of IC containing modified LDL is unique to patients with diabetes. The fact that antibodies to modified LDL have also been detected and isolated from non-diabetic patients with coronary heart disease and healthy volunteers (Virella et al., 1993, 2002) and that IC complexes prepared with human oxLDL and human oxLDL antibodies isolated from patients with diabetes and healthy volunteers have similar pro-atherogenic and pro-inflammatory properties (Virella et al., 2002) argues in favor of a general role of LDLIC in the pathogenesis of atherosclerosis. On the other hand, IC isolated from patients with type 2 diabetes and macrovascular disease are enriched in cholesterol and apolipoprotein B and induce significantly higher accumulation of cholesterol than IC isolated from non-diabetic patients with coronary artery disease or from healthy volunteers (Mironova et al., 2000). This could reflect an enhanced generation of modified LDL in diabetes, due to the increased oxidative stress, and to a lower content of antioxidants in the LDL isolated from diabetic patients (Mironova et al., 2000). An increased antigenic load would lead to a more vigorous immune response and to higher levels of IC containing modified LDL, thus resulting in a more prominent pathogenic role in diabetes.

\section{MODIFIED FORMS OF LDL AS BIOMARKERS OF PLAQUE INSTABILITY}

There is considerable interest in identifying biomarkers indicative of plaque instability. A variety of proteins and enzymes have been proposed as candidates, as reviewed recently by Koenig and Khuseyinova (2007). Besides MMPs, C-reactive proteins (CRP), cytokines (IL-6, IL-18), enzymes [glutathione peroxidase, lipoprotein-associated phospholipase A2 (Lp-PLA2), type II secretory phospholipase A2, myeloperoxidase, MMPs, particularly MMP-9, and pregnancy-associated plasma protein A], chemotactic proteins (monocyte chemotactic protein-1), placental growth factor, soluble CD40 ligand, soluble Fas ligand, and oxLDL have been proposed as indicators of plaque instability (Holvoet et al., 1995, 1998; Holvoet, 1999; Loftus et al., 2001; Pelisek et al., 2009; Colley et al., 2011). CRP has been extensively studied and several studies support the correlation between CRP levels and risk to suffer an acute cardiovascular event, but its predictive power beyond the traditional risk factors remains unproven (Koenig and Khuseyinova, 2007). Similar uncertainties, often compounded by lack of precise measuring methods, apply to most other proposed indicators of plaque instability (Koenig and Khuseyinova, 2007).

Of these markers, MDA-LDL and MDA-LDL-IC, oxLDL-IC, Lp-PLA2, TIMPs, and MMPs appear the most likely to have clinical 
relevance, given the results obtained in preliminary clinical studies and/or their biological properties that are directly related plaque instability (Holvoet et al., 1995, 1998; Holvoet, 1999; Loftus et al., 2001; Koenig and Khuseyinova, 2007; Lopes-Virella et al., 2007, 2011a,b, 2012b; Pelisek et al., 2009; Colley et al., 2011). However, most studies have been carried out in small patient populations, and the results are not consistent. Our studies in the DCCT/EDIC and VADT cohorts of patients with type 1 and type 2 diabetes are exceptions to this rule, and have demonstrated significant associations not only with cardiovascular disease (Lopes-Virella et al., 2007, 2011a,b), but also with nephropathy (Lopes-Virella et al., 2012a) and retinopathy (Lopes-Virella et al., 2012c). This high predictive power of the measurement of $\mathrm{mLDL}$ in IC reflects the fact that our assay allows the accurate measurement of the levels of different modifications of the LDL molecules contained

\section{REFERENCES}

Al Gadban, M. M., Smith, K. J., Soodavar, F., Piansay, C., Chassereau, C., Twal, W. O., Klein, R. L., Virella, G., Lopes-Virella, M. F., and Hammad, S. M. (2010). Differential trafficking of oxidized LDL and oxidized LDL immune complexes in macrophages: impact on oxidative stress. PLoS ONE 5, el2534. doi:10.1371/journal.pone.0012534

Andersson, J., Libby, P., and Hansson, G. K. (2010). Adaptive immunity and atherosclerosis. Clin. Immunol. 134, 33-46.

Arai, K., Kita, T., Yokode, M., Narumiya, S., and Kawai, C. (1989). Multiple receptors for modified LDL in mouse peritoneal macrophages: different uptake mechanisms for acetylated and oxidized LDL. Biochem. Biophys. Res. Commun. 159, 1375-1379.

Atchley, D. H., Lopes-Virella, M. F., Zheng, D., and Virella, G. (2002). Oxidized LDL-anti-oxidized LDL immune complexes and diabetic nephropathy. Diabetologia 45, 1562-1571.

Berliner, J. A., Territo, M. C., Sevanian, A., Raimin, S., Kim, J. A., Bamshad, B., Ester-son, M., and Fogelman, A. M. (1990). Minimally modified low density lipoprotein stimulates monocyte endothelial interactions. J. Clin. Invest. 85, 1260-1266.

Blankenberg, S., Rupprecht, H. J., Poirier, O., Bickel, C., Smieja, M., Hafner, G., Meyer, J., Cambien, F., and Tiret, L. (2003). Plasma concentrations and genetic variation of matrix metalloproteinase 9 and prognosis of patients with cardiovascular disease. Circulation 107, 1579-1585.

Bucciarelli, L. G., Wendt, T., Qu, W., Lu, Y., Lalla, E., Rong, L. L., Goova, M. T., Moser, B., Kislinger, T., Lee, D. C., Kashyap, Y., Stern, D. M., and Schmidt, A. M. (2002). RAGE blockade stabilizes established atherosclerosis in diabetic apolipoprotein E-null mice. Circulation 106, 2827-2835

Chen, J. H., Riazy, M., Wang, S. W. brecher, U. P. (2010). Sphingosine kinase regulates oxidized low density lipoprotein-mediated calcium oscillations and macrophage survival. $J$. Lipid Res. 51, 991-998.

Choi, S. H., Harkewicz, R., Lee, J. H., Boullier, A., Almazan, F., Li, A. C., Witztum, J. L., Bae, Y. S., and Miller, Y. I. (2009). Lipoprotein accumulation in macrophages via toll-like receptor-4-dependent fluid phase uptake. Circ. Res. 104, 1355-1363.

Colley, K. J., Wolfert, R. L., and Cobble, M. E. (2011). Lipoprotein associated phospholipase $\mathrm{A}(2)$ : role in atherosclerosis and utility as a biomarker for cardiovascular risk. EPMA J. 2, 27-38.

Crowley, M. T., Costello, P. S., FitzerAttas, C. J., Turner, M., Meng, F., Lowell, C., Tybulewicz, V. L., and DeFranco, A. L. (1997). A critical role for Syk in signal transduction and phagocytosis mediated by Fcgamma receptors on macrophages. J. Exp. Med. 186, 1027-1039.

Datta, S. R., Brunet, A., and Greenberg, M. E. (1999). Cellular survival: a play in three Akts. Genes Dev. 13, 2905-2927.

de Boer, O. J., van der Wal, A. C., Houtkamp, M. A., Ossewaarde, J. M., Teeling, P., and Becker, A. E. (2000). Unstable atherosclerotic plaques contain T-cells that respond to Chlamydia pneumoniae. Cardiovasc. Res. 48, 402-408.

De Boer, O. J., van der Wal, A. C., Verhagen, C. E., and Becker, A. E. Dai, J. M., Duronio, V., and Stein(1999). Cytokine secretion profiles

in IC (Virella and Lopes-Virella, 2003; Virella et al., 2005). This is extremely significant, because, as previously mentioned, $95 \%$ or more of the modified LDL in circulation is associated with the corresponding antibodies forming IC (Virella et al., 2004), and because IC containing modified LDL are considerably more pathogenic than modified LDL by itself as consequence of their ability to engage $\mathrm{F} c \gamma$ receptors and activate phagocytic cells to a much greater extent (Virella et al., 2002; Saad et al., 2006). Ongoing studies will further define the predictive value of the analysis of the content of mLDL in circulating IC, which at the present time appears to have the potential to become the best predictor of progression and complications of cardiovascular disease in diabetic patients. Also in the planning stages are studies aimed at proving that the same predictive value will extend to non-diabetic populations.

of cloned $\mathrm{T}$ cells from human aortic atherosclerotic plaques. J. Pathol. 188, 174-179.

Endemann, G., Stanton, L. W., Madden, K. S., Bryant, C. M., White, R. T., and Protter, A. A. (1993). CD36 is a receptor for oxidized LDL. J. Biol. Chem. 268, 11811-11818.

Fogelman, A. M., Schechter, I., Seager, J., Hokom, M., Child, J. S., and Edwards, P. A. (1980). Malondialdehyde alteration of low density lipoproteins leads to cholesterol ester accumulation in human monocytes/macrophages. Proc. Natl. Acad. Sci. U.S.A. 77, 2214-2218.

Galis, Z. S., Sukhova, G. K., Lark, M. W., and Libby, P. (1994). Increased expression of matrix metalloproteinases and matrix degrading activity in vulnerable regions of human atherosclerotic plaques. $J$. Clin. Invest. 94, 2493-2503.

Giacco, F., and Brownlee, M. (2010). Oxidative stress and diabetic complications. Circ. Res. 107, 1058-1070.

Hammad, S. M., Taha, T. A., Nareika, A., Johnson, K. R., Lopes-Virella, M. F., and Obeid, L. M. (2006). Oxidized LDL immune complexes induce release of sphingosine kinase in human U937 monocytic cells. Prostaglandins Lipid Mediat. 79, 126-140.

Hammad, S. M., Twal, W. O., Barth, J. L., Smith, K. J., Saad, A. F., Virella, G., Argraves, W. S., and LopesVirella, M. F. (2009). Oxidized LDL immune complexes and oxidized LDL differentially affect the expression of genes involved with inflammation and survival in human U937 monocytic cells. Atherosclerosis 202, 394-404.

Henriksen, T., Evensen, S. A., and Carlander, B. (1979). Injury to human endothelial cells in culture induced by LDL. Scand. J. Clin. Lab. Invest. 39, 361-364.
Henriksen, T., Mahoney, M. E., and Steinberg, D. (1983). Enhanced macrophage degradation of biologically modified low density lipoprotein. Arteriosclerosis 3, 149-158.

Hessler, J. R., Morel, D. W., Lewis, L. J., and Chisolm, G. M. (1983). Lipoprotein oxidation and lipoproteininduced cytotoxicity. Arteriosclerosis 3, 215-222.

Hoff, H. F., O’Neil, J., Chisolm, G. M., Cole, T. B., Quehenberger, O., Esterbauer, H., and Jurgens, G. (1989). Modification of low density lipoprotein with 4-hydroxynonenal induces uptake by macrophages. Arteriosclerosis 9, 538-549.

Hoff, H. F., Zyromski, N., Armstrong, D., and O’Neil, J. (1993). Aggregation as well as chemical modification of LDL during oxidation is responsible for poor processing in macrophages. J. Lipid Res. 34, 1919-1929.

Holvoet, P. (1999). Endothelial dysfunction, oxidation of low-density lipoprotein, and cardiovascular disease. Ther. Apher. 3, 287-293.

Holvoet, P., Perez, G., Zhao, Z., Brouwers, E., Bernar, H., and Collen, D. (1995). Malondialdehyde-modified low density lipoproteins in patients with atherosclerotic disease. J. Clin. Invest. 95, 2611-2619.

Holvoet, P., Vanhaecke, J., Janssens, S., Van de Werf, F., and Collen, D. (1998). Oxidized LDL and malondialdehyde-modified LDL in patients with acute coronary syndromes and stable coronary artery disease. Circulation 98, 1487-1494.

Hundal, R. S., Gomez-Munoz, A., Kong, J. Y., Salh, B. S., Marotta, A., Duronio, V., and Steinbrecher, U. P. (2003). Oxidized low density lipoprotein inhibits macrophage apoptosis by blocking ceramide generation, thereby maintaining protein kinase B activation and Bcl-XL levels. J. Biol. Chem. 278, 24399-24408. 
Jarrett, R. J. (1981). Atherosclerosis, diabetes and obesity. Proc. Nutr. Soc. 40, 209-212.

Kahn, B. V., Parthasarathy, S. S., Alexander, R. W., and Medford, R. M. (1995). Modified LDL and its constituents augment cytokineactivated vascular cell adhesion molecule-1 gene expression in human vascular endothelial cells. $J$. Clin. Invest. 95, 1262-1270.

Koenig, W., and Khuseyinova, N. (2007). Biomarkers of atherosclerotic plaque instability and rupture. Arterioscler. Thromb. Vasc. Biol. 27, 15-26.

Kugiyama, K., Sakamoto, T., Musumi, I., Sugiyama, S., Ohgushi, M., Ogawa, H., Horiguchi, M., and Yasue, $\mathrm{H}$. (1993). Transferrable lipids in oxidized LDL stimulate PAI-1 and inhibit tPA release from endothelial cells. Circ. Res. 73, 335-343.

Kume, N., Cybulsky, M. I., and Gimbrone, M. A. J. (1992). Lysophosphatidyl-choline, a component of atherogenic lipoproteins, induces mononuclear leukocyte adhesion molecules in cultured human and rabbit arterial endothelial cells. J. Clin. Invest. 90, 1138-1144.

Langer, T., Strober, W., and Levy, R. I. (1972). The metabolism of low density lipoprotein in familial type II hyperlipoproteinemia. J. Clin. Invest. 51, 1528-1536.

Li, W., Febbraio, M., Reddy, S. P., Yu, D. Y., Yamamoto, M., and Silverstein, R. L. (2010). CD36 participates in a signaling pathway that regulates ROS formation in murine VSMCs. J. Clin. Invest. 120, 3996-4006.

Libby, P., and Theroux, P. (2005). Pathophysiology of coronary artery disease. Circulation 111, 3481-3488.

Lim, W. S., Timmins, J. M., Seimon, T. A., Sadler, A., Kolodgie, F. D., Virmani, R., and Tabas, I. (2008). Signal transducer and activator of transcription-1 is critical for apoptosis in macrophages subjected to endoplasmic reticulum stress in vitro and in advanced atherosclerotic lesions in vivo. Circulation 117, 940-951.

Loftus, I. M., Naylor, A. R., Bell, P. R., and Thompson, M. M. (2001). Plasma MMP-9 - a marker of carotid plaque instability. Eur. J. Vasc. Endovasc. Surg. 21, 17-21.

Lopes-Virella, M. F., Binzafar, N., Rackley, S., Takei, A., La Via, M., and Virella, G. (1997). The uptake of LDL-IC by human macrophages: predominant involvement of the Fc gamma RI receptor. Atherosclerosis 135, 161-170.
Lopes-Virella, M. F., Griffith, R. L., Shunk, K. A., and Virella, G. T. (1991). Enhanced uptake and impaired intracellular metabolism of low density lipoprotein complexed with anti-low density lipoprotein antibodies. Arterioscl. Thromb. 11, 1356-1367.

Lopes-Virella, M. F., Hunt, K. J., Baker, N. L., Lachin, J., Nathan, D. M., and Virella, G. (2011a). The levels of oxidized ldl and age- ldl in circulating immune complexes are strongly associated with increased levels of carotid intima-media thickness and its progression in type 1 diabetes. Diabetes 60, 582-589.

Lopes-Virella, M. F., Baker, N. L., Hunt, K. J., Lachin, J., Nathan, D., and Virella, G. (2011b). Oxidized LDL immune complexes and coronary artery calcification in type 1 diabetes. Atherosclerosis 214, 462-467.

Lopes-Virella, M. F., Carter, R. E., Baker, N. L., Lachin, J., Virella, G., and Group, D. E. R. (2012a). High levels of oxidized LDL in circulating immune complexes are associated with increased odds of developing abnormal albuminuria in Type 1 diabetes. Nephrol. Dial. Transplant. 27, 1416-1423.

Lopes-Virella, M. F., Hunt, K. J., and Baker, N. L., Moritz, T., Virella, G. and The VADT Group of Investigators. (2012b). Levels of MDA LDL in circulating immune complexes predict myocardial infarction in the VADT study in the Veterans Affairs Diabetes Trial (VADT). J. Clin. Lipidol. 6, 256.

Lopes-Virella, M. F., Baker, N. L., Hunt, K. J., Lyons, T. J., Jenkins, A. J., Virella, G., and The DCCT/EDIC Study Group. (2012c). The concentration of age-modified LDL and oxidized LDL in circulating immune complexes is associated with progression of retinopathy in type 1 diabetes. Diabetes Care 35, 1333-1340.

Lopes-Virella, M. F., McHenry, M. B., Lipsitz, S., Yim, E., Wilson, P. F., Lackland, D. T., Lyons, T., Jenkins, A. J., and Virella, G. (2007). Immune complexes containing modified lipoproteins are related to the progression of internal carotid intima-media thickness in patients with type 1 diabetes. Atherosclerosis 190, 359-369.

Lopes-Virella, M. F., and Virella, G. (2003). The role of immune and inflammatory processes in the development of macrovascular disease in diabetes. Front. Biosci. 8, s750-s768. Lopes-Virella, M. F., and Virella, G. (2010). Clinical significance of the humoral immune response to modified LDL. Clin. Immunol. 134, 55-65.

Lundberg, A. M., and Hansson, G. K. (2010). Innate immune signals in atherosclerosis. Clin. Immunol. 134 5-24.

Luo, Y., Pollard, J. W., and Casadevall, A. (2010). Fcgamma receptor crosslinking stimulates cell proliferation of macrophages via the ERK pathway. J. Biol. Chem. 285, 4232-4242.

Miller, Y. I., Choi, S. H., Fang, L., and Tsimikas, S. (2010). Lipoprotein modification and macrophage uptake: role of pathologic cholesterol transport in atherogenesis. Subcell. Biochem. 51, 229-251.

Mironova, M., Virella, G., and LopesVirella, M. F. (1996). Isolation and characterization of human antioxidized LDL autoantibodies. Arterioscler. Thromb. Vasc. Biol. 16, 222-229.

Mironova, M. A., Klein, R. L., Virella, G. T., and Lopes-Virella, M. F. (2000). Anti-modified LDL antibodies, LDL-containing immune complexes, and susceptibility of LDL to in vitro oxidation in patients with type 2 diabetes. Diabetes 49 , 1033-1041.

Moreno, P. R., Murcia, A. M., Palacios, I. F., Leon, M. N., Bernardi, V. H., Fuster, V., and Fallon, J. T. (2000). Coronary composition and macrophage infiltration in atherectomy specimens from patients with diabetes mellitus. Circulation 102, 2180-2184.

Nagarajan, S. (2007). Anti-OxLDL IgG blocks OxLDL interaction with CD36, but promotes FcgammaR, CD32A-dependent inflammatory cell adhesion. Immunol. Lett. 108, 52-61.

Oksjoki, R., Kovanen, P. T., Lindstedt, K. A., Jansson, B., and Pentikainen, M. O. (2006). OxLDL-IgG immune complexes induce survival of human monocytes. Arterioscler. Thromb. Vasc. Biol. 26, 576-583.

Orekhov, A. N., Kalenich, O. S., Tertov, V. V., and Novikov, I. D. (1991). Lipoprotein immune complexes as markers of atherosclerosis. Int. J. Tissue React. 13, 233-236.

Orekhov, A. N., Kalenich, O. S., Tertov, V. V., Perova, N. V., Novikov Iy, D., Lyakishev, A. A., Deev, A. D., and Ruda, M. (1995). Diagnostic value of immune cholesterol as a marker for atherosclerosis. J. Cardiovasc. Risk 2 , 459-466.

Palinski, W., Yla-Herttuala, S., Rosenfeld, M. E., Butler, S. W., Socher, S. A., Parthasarathy, S., Curtiss, L. K., and Witztum, J. L. (1990). Antisera and monoclonal antibodies specific for epitopes generated during oxidative modification of low density lipoprotein. Arteriosclerosis 10, 325-335.

Pelisek, J., Rudelius, M., Zepper, P., Poppert, H., Reeps, C., Schuster, T., and Eckstein, H. H. (2009). Multiple biological predictors for vulnerable carotid lesions. Cerebrovasc. Dis. 28, 601-610.

Penn, M. S., and Chisolm, G. M. (1994). Oxidized lipoproteins, altered cell function and atherosclerosis. Atherosclerosis 108, S21-S29.

Quinn, M. T., Parthasarathy, S., Fong, L. G., and Steinberg, D. (1987). Oxidatively modified low density lipoproteins: a potential role in recruitment and retention of monocyte/macrophages during atherogenesis. Proc. Natl. Acad. Sci. U.S.A. 84, 2995-2998.

Rajavashisth, T. B., Andalibi, A., and Territo, M. C. (1990). Induction of endothelial cell expression of granulocyte and macrophage colonystimulating factors by modified LDL. Nature 344, 254-257.

Saad, A. F., Virella, G., Chassereau, C., Boackle, R. J., and LopesVirella, M. F. (2006). OxLDL immune complexes activate complement and induce cytokine production by MonoMac 6 cells and human macrophages. J. Lipid Res. 47: 1975-1983.

Schmidt, A. M., Hori, O., Chen, J. X., Li, J. F., Crandall, J., Zhang, J., Cao, R., Yan, S. D., Brett, J., and Stern, D. (1995). Advanced glycation endproducts interacting with their endothelial receptor induce expression of vascular cell adhesion molecule-1 (VCAM-1) in cultured human endothelial cells and in mice. A potential mechanism for the accelerated vasculopathy of diabetes. $J$. Clin. Invest. 96, 1395-1403.

Seimon, T., and Tabas, I. (2009). Mechanisms and consequences of macrophage apoptosis in atherosclerosis. J. Lipid Res. 50(Suppl.), S382S387.

Shah, P. K. (2002). Pathophysiology of coronary thrombosis: role of plaque rupture and plaque erosion. Prog. Cardiovasc. Dis. 44, 357-368.

Shalhoub, J., Falck-Hansen, M. A., Davies, A. H., and Monaco, C. (2011). Innate immunity and monocyte-macrophage activation in atherosclerosis. J. Inflamm. (Lond.) 8, 9.

Silverstein, R. L. (2009). Type 2 scavenger receptor CD36 in platelet activation: the role of hyperlipemia and oxidative stress. Clin. Lipidol. 4, 767.

Silverstein, R. L., Li, W., Park, Y. M., and Rahaman, S. O. (2010). 
Mechanisms of cell signaling by the scavenger receptor CD36: implications in atherosclerosis and thrombosis. Trans. Am. Clin. Climatol. Assoc. 121, 206-220.

Smith, K. J., Twal, W. O., Soodavar, F., Virella, G., Lopes-Virella, M. F., and Hammad, S. M. (2010). Heat shock protein 70B' (HSP70B') expression and release in response to human oxidized low density lipoprotein immune complexes in macrophages. J. Biol. Chem. 285, 15985-15993.

Sparrow, C. P., Parthasarathy, S., and Steinberg, D. (1989). A macrophage receptor that recognizes oxidized low density lipoprotein but not acetylated low density lipoprotein. J. Biol. Chem. 264, 2599-2604.

Steinbrecher, U. P. (1987). Oxidation of human low density lipoprotein results in derivatization of lysine residues of apolipoprotein B by lipid peroxide decomposition products. J. Biol. Chem. 262, 3603-3608.

Steinbrecher, U. P., Fisher, M., Witztum, J. L., and Curtiss, L. K. (1984). Immunogenicity of homologous low density lipoprotein after methylation, ethylation, acetylation, or carbamylation: generation of antibodies specific for derivatized lysine. J. Lipid Res. 25, 1109-1116.

Stewart, C. R., Stuart, L. M., Wilkinson, K., van Gils, J. M., Deng, J., Halle, A., Rayner, K. J., Boyer, L., Zhong, R., Frazier, W. A., Lacy-Hulbert, A., El Khoury, J., Golenbock, D. T., and Moore, K. J. (2010). CD36 ligands promote sterile inflammation through assembly of a Toll-like receptor 4 and 6 heterodimer. Nat. Immunol. 11, 155-161.
Takei, A., Huang, Y., and Lopes-Virella, M. F. (2001). Expression of adhesion molecules by human endothelial cells exposed to oxidized low density lipoprotein. Influences of degree of oxidation and location of oxidized LDL. Atherosclerosis 154 79-86.

Tohyama, Y., and Yamamura, H. (2009). Protein tyrosine kinase, syk: a key player in phagocytic cells. J. Biochem. 145, 267-273.

Viita, H., Narvanen, O., and YlaHerttuala, S. (1999). Different apolipoprotein B breakdown patterns in models of oxidized low density lipoprotein. Life Sci. 65, 783-793.

Virella, G. (2007). "Biosynthesis, metabolism and biological properties of immunoglobulins," in Medical Immunology, ed. G. Virella (New York: Informa), 65-72.

Virella, G., Atchley, D. H., Koskinen, S., Zheng, D., and LopesVirella, M. (2002). Pro-atherogenic and pro-inflammatory properties of immune complexes prepared with purified human oxLDL antibodies and human oxLDL. Clin. Immunol. 105, 81-92.

Virella, G., Carter, R. E., Saad, A., Crosswell, E. G., Game, B. A., and LopesVirella, M. F. (2008). Distribution of IgM and IgG antibodies to oxidized LDL in immune complexes isolated from patients with type 1 diabetes and its relationship with nephropathy. Clin. Immunol. 127, 394-400.

Virella, G., Derrick, M. B., Pate, V., Chassereau, C., Thorpe, S. R., and Lopes-Virella, M. F. (2005).
Development of capture assays for different modifications of human low-density lipoprotein. Clin. Diagn Lab. Immunol. 12, 68-75.

Virella, G., Koskinen, S., Krings, G., Onorato, J. M., Thorpe, S. R., and Lopes-Virella, M. (2000). Immunochemical characterization of purified human oxidized lowdensity lipoprotein antibodies. Clin. Immunol. 95, 135-144.

Virella, G., and Lopes-Virella, M. F. (2003). Lipoprotein autoantibodies: measurement and significance. Clin. Diagn. Lab. Immunol. 10 499-505.

Virella, G., Thorpe, S., Alderson, N. L., Derrick, M. B., Chassereau, C., Rhett, J. M., and Lopes-Virella, M. F. (2004). Definition of the immunogenic forms of modified human LDL recognized by human autoantibodies and by rabbit hyperimmune antibodies. J. Lipid Res. 45, 1859-1867.

Virella, G., Virella, I., Leman, R. B. Pryor, M. B., and Lopes-Virella, M. F. (1993). Anti-oxidized low-density lipoprotein antibodies in patients with coronary heart disease and normal healthy volunteers. Int. J. Clin. Lab. Res. 23, 95-101.

Vlassara, H., Bucala, R., and Striker L. (1994). Pathogenic effects of advanced glycosylation: biochemical, biologic, and clinical implications for diabetes and aging. Lab. Invest. 70, 138-151.

Vlassara, H., Cai, W., Crandall, J. Goldberg, T., Oberstein, R., Dardaine, V., Peppa, M., and Rayfield, E. J. (2002). Inflammatory mediators are induced by dietary glycotoxins, a major risk factor for diabetic angiopathy. Proc. Natl. Acad. Sci. U.S.A. 99, 15596-15601.

Wendt, T., Bucciarelli, L., Qu, W., Lu, Y., Yan, S. F., Stern, D. M., and Schmidt, A. M. (2002). Receptor for advanced glycation endproducts (RAGE) and vascular inflammation: insights into the pathogenesis of macrovascular complications in diabetes. Curr. Atheroscler. Rep. 4, 228-237.

Yla-Herttuala, S., Palinski, W., Butler, S., Picard, S., Steinberg, D., and Witztum, J. L. (1994). Rabbit and human atherosclerotic lesions contain IgG that recognizes epitopes of oxidized LDL. Arterioscler. Thromb. 14, 32-40.

Conflict of Interest Statement: The authors declare that the research was conducted in the absence of any commercial or financial relationships that could be construed as a potential conflict of interest.

Received: 05 April 2012; accepted: 28 May 2012; published online: 15 June 2012.

Citation: Virella G and Lopes-Virella MF (2012) The pathogenic role of the adaptive immune response to modified LDL in diabetes. Front. Endocrin. 3:76. doi: 10.3389/fendo.2012.00076

This article was submitted to Frontiers in Diabetes, a specialty of Frontiers in Endocrinology.

Copyright (C) 2012 Virella and LopesVirella. This is an open-access article distributed under the terms of the Creative Commons Attribution Non Commercial License, which permits noncommercial use, distribution, and reproduction in other forums, provided the original authors and source are credited. 